\title{
Beyond coloured balls - making sense of large volumes of microseismic data with diverse source mechanisms
}

\author{
W. de Beer Golder Associates (NZ) Ltd, New Zealand
}

J.A. Ardito MINSUR S.A., Peru

J-P. Mercier Golder Associates Ltd., Canada; and Advanced Geosciences Imaging Solutions Ltd., Canada

V. Falmagne Golder Associates Ltd., Canada

\section{Abstract}

The central theme of this analysis is the study of seismic response of the rock mass before and after the occurrence of a moment magnitude 2.0 seismic event that occurred in December 2010 at the San Rafael Mine sublevel open stoping operation located in Peru. A microseismic data set acquired by the mine microseismic monitoring system is analysed using a range of methodologies, including passive source tomography imaging, focal mechanism inversion, as well as deterministic and probabilistic approaches. $P$ - and S-wave velocity models, calculated for three time periods, using a passive source tomography algorithm developed by Advanced GeoScience Imaging Solutions (AGSIS), was applied to the data set to image the evolution of the rock mass. The models show variations in the seismic velocity before and after the event that may relate to the distribution and redistribution of stresses in the rock mass. Probable focal mechanisms, exploration of the spatial geometry of seismic event distributions (using singular value decomposition and clustering) as well as rapid separation of populations of events which are generated by different source mechanisms were also used to illuminate different aspects of the seismic response to mining at that time. We note that the separation of populations serves only to provide consistent and unique distributions to use in the assessment of hazard due to particular types of activity. The complete dataset (with the possible exception of blasts and ore pass noise) is required for modelling velocity structures and understanding stress transfer mechanisms in the rock mass.

\section{Introduction}

On 17 December 2010 a seismic event of Moment Magnitude $M_{w} 2.0$ occurred on San Rafael Mine. The shaking was felt and heard on the surface and significant damage to mine infrastructure and excavations was reported as far as $130 \mathrm{~m}$ away from the event hypocenter. Conventional analysis of the data (comparison of seismicity with blasting and production, trends in stress and strain related seismic parameters) do not account for areas of the rock mass which, although there may not be seismic activity, nevertheless are traversed by seismic energy. We will illustrate that these traditional methods also require considerable effort in filtering and separation. The back-analysis of this event provided an opportunity for exploiting the properties of a dense data set in two different but complementary ways, thereby correlating passive seismic tomography and conventional analyses.

The microseismic monitoring system at San Rafael records a large data sets, enabling a variety of advanced seismic analyses. Approximately $10 \%$ of these events are friction-dominated ruptures (slip-dominant, 'conventional' events) and the rest form a mix of blasts, orepass noise, and pillar crushing and fracturing of intact rock immediately after blasting. Using the principle that all sources of elastic waves serve to illuminate the rock mass, we wish to avoid denuding the data for the sake of visualising a comprehensible collection of coloured balls. Rather, we propose embracing the qualities of the data to progress towards mapping the changes in the underlying properties of the rock mass in a manner consistent with the results of conventional analyses. In this paper we show that the density of the data set presents opportunities for 
both high-resolution conventional analysis and for applying passive seismic tomography which requires such density and which have become feasible with advances in computer technology.

We performed the back analysis by considering three months of seismic activity before and after the event. Probabilistic hazard assessment requires statistical distributions with unambiguous critical exponents; for this reason the different types of events had to be separated and we illustrate a rapid separation method based on clustering, using corner frequency as a discrimination metric. Spatial clustering applied to the separated event types is used to enumerate particular seismicity sources for the purposes of deterministic hazard assessment.

The tomography results were found to be in broad agreement with trends in the conventional analysis. Parallels with laboratory results suggest that the behaviour of the tomography-derived velocity model could be indicative of the changing stress state of the rock mass.

\section{1 Overview of San Rafael Mine}

The San Rafael Mine is a steeply-dipping, north-northwest/west-southwest striking, high grade, lode-type $\mathrm{Sn}-\mathrm{Cu}$ deposit in the Eastern Cordillera of the Peruvian Central Andes. The bulk of the tin mineralisation is hosted by a leucomonzogranite. The lode is mined by sublevel open stoping, with levels developed from a decline or ramp which spirals down predominantly in the footwall. The of interest area stretches from approximately chainage 1,850 to $2,150 \mathrm{~m}$ (centred on chainage $2,000 \mathrm{~m}$ ) along the long section, and from level (nv.) 3850 to 4310.

\section{2 Overview of the San Rafael microseismic monitoring system}

The San Rafael microseismic monitoring system consists of an array (Figure 1) of 24 Sensor Nederland SM-6 geophone elements connected to four ISS International/IMS GS seismometers. The geophone elements are housed in eight ensembles of three mutually orthogonal elements (thus making up eight triaxial geophone sites) distributed in such a way as to include the hangingwall and footwall of an area of the mine of particular interest. The region of high confidence is a volume of rock which is predominantly inside the network, where it could be expected that the uncertainty in hypocentre determination would be the lowest.

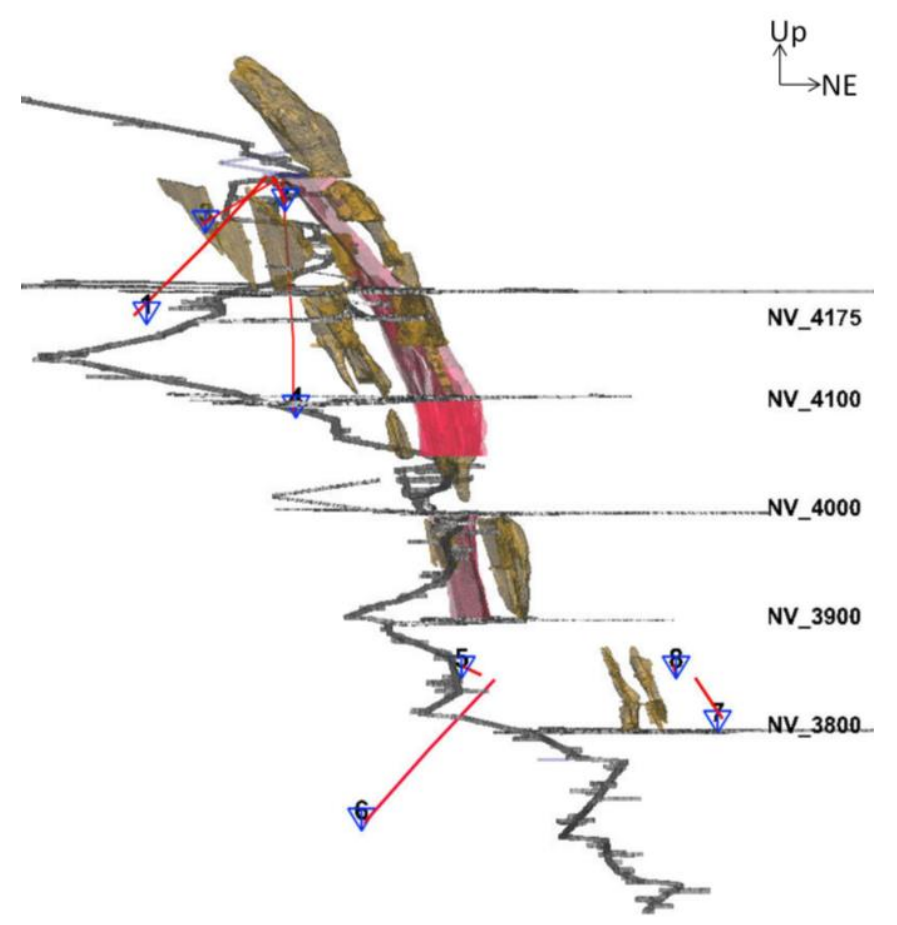

Figure 1 Geometry of the sensor array, view along strike to the northwest 
The data acquisition system was commissioned from by the Institute of Mine Seismology (IMS) and was installed in September 2010. Software enhancements extend the flat frequency response region of the SM-6 geophone down to approximately $8 \mathrm{~Hz}$ (R. Lynch, 2011, written comm.).

\section{3 Overview of $M_{w} 2.0$ event of December 2010}

\subsection{Location and properties}

The $M_{w} 2.0$ 2010/12/17, 07:17:09 PET event (Figure 2) occurred at mine coordinates Easting $(X)=$ E356,916 m, Northing $(\mathrm{Y})=\mathrm{N} 8,429,085 \mathrm{~m}$, Elevation $(\mathrm{RL}$ or $\mathrm{Z})$ ) $=\mathrm{RL4,043} \mathrm{m}$, with an estimated uncertainty in the location of $\pm 8 \mathrm{~m}$.

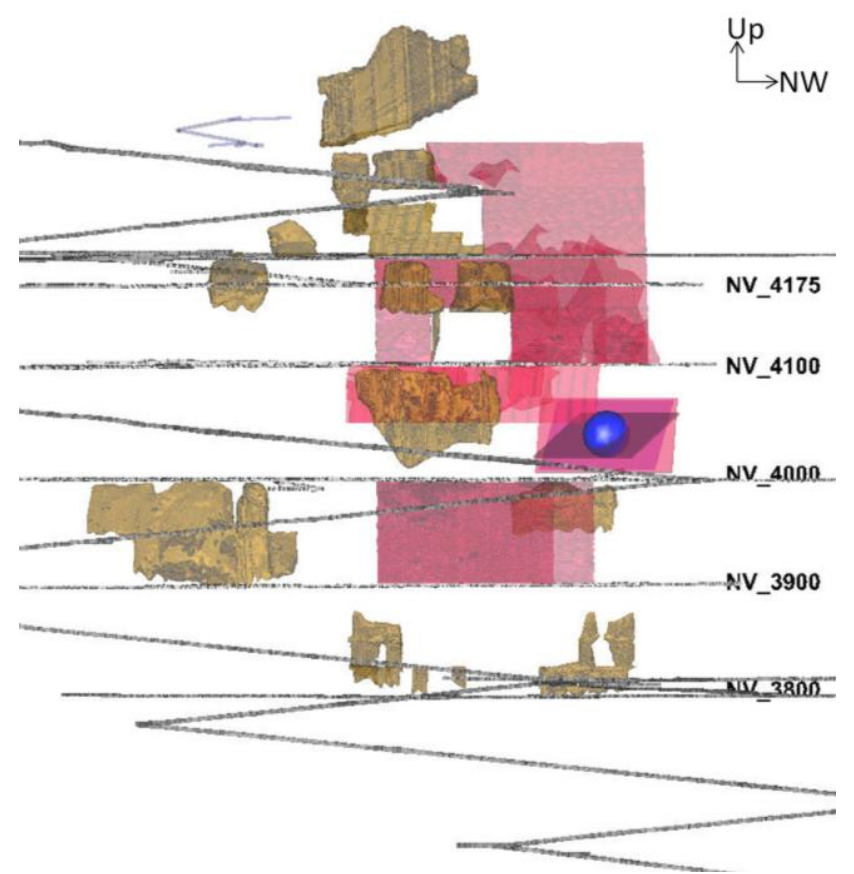

Figure 2 Location and probable fault planes of the 2010/12/17, 07:17:09 PET event

The location of the event relative to those sensors in the array used to calculate its properties was such that it is not possible to determine a single definitive failure mechanism. Nevertheless, three probable solutions are strike-slip along a dipping plane, oblique-reverse or strike-slip with oblique motion, ranging from strike $356^{\circ}$ (steeply dipping at $80^{\circ}$ ) to $11^{\circ}$ (steeply dipping at $67^{\circ}$ ).

\subsection{Damage}

Ground motion and noise were felt and heard on surface at the time of the event. Underground, significant damage was done to Stope T4100-01 on NV 4065, in an area centred on a point approximately $50 \mathrm{~m}$ southeast and $24 \mathrm{~m}$ above the calculated location of the event. The effects of the event in Stope T4100-01 on NV 4065 have been variously described as "rockburst" and "collapse". Further to the southeast, on NV 4075, a concrete fan support was blown off its foundations. On NV 5175 (approximately $130 \mathrm{~m}$ above the calculated location of the event, significant floor damage was incurred.

\section{3. 3 Seismic context}

The $M_{w} 2.0$ 2010/12/17, 07:17:09 PET event appears to have been be the conclusion of a sequence of seismic activity which delineated a planar feature in the spatial distribution of activity. The feature is visible in the data set from 1 November 2010 through January 2011, but is at its most prominent in the period before 26 November 2010. 
Fitting a plane through the locations of some of the seismic events in the period 1 November 2010 to 26 November 2010 gives a plane centred on E356,916 m, N8,429,085 m RL4,043 m, with a length of $122 \mathrm{~m}$, width $91 \mathrm{~m}$, strike $180^{\circ}$ and dip $39^{\circ}$.

\subsection{Mining context}

The $M_{w} 2.0$ 2010/12/17, 07:17:09 PET event occurred in a period in which the number of blasts was lower than corresponding periods; however the total tonnage blasted in the five days before the event was similar or higher to other five-day periods. Most of it appeared to be concentrated on 15 December 2010.

There was no or very little blasting on 13 and 14 December 2010, followed by substantial blasting on 15 December 2010. 16 December 2010 again saw no blasting.

\section{Methodology}

The $M_{w} 2.0$ 2010/12/17, 07:17:09 PET event was considered against the background of three months of seismicity, from 1 November 2010 to 31 January 2011, the event occurring roughly in the middle of this period. Two complementary methods of data analysis were followed: application of a passive source tomography analysis and a conventional spatiotemporal approach.

For the passive source tomography analysis, all the data are utilised, including rejected events. In this way, all sources of acoustic emission are utilised, with the manually processed and accepted events used as a consistency check. Seismic tomography is a standard geophysical imaging method often used to infer geotechnical characteristics of rock by calculating and mapping seismic velocities in a given material. Passive source tomography uses seismic energy from natural and induced seismicity which traverses the rock mass. The key is that even regions which may at a given time be seismically inactive could be illuminated by seismic energy from distant sources. Laboratory scale studies have shown relationships between seismic velocities, stress and strain state and mechanical properties of rock, e.g. Guéguen et al. (2009) and Angus et al. (2009).

Conventional analysis of seismicity can be summarised in a few steps: identification of sources of seismicity (these are collections of seismic events), investigation of the statistical properties (including the occurrence and likelihood of damaging events) of a source volume, investigation of trends in stress and strain-release related seismic parameters and, finally, proposition of likely causes for the seismic rock mass response in a given source area.

The first two require an additional step, which is to identify different source types. Of these, the first step is crucial: Seismicity does not occur in hard-walled containers - seismic source volumes are clusters of events with diffuse and irregular borders. It is therefore necessary to apply an objective method to delineate source volumes (Hudyma, 2008). The investigation of statistical properties requires unique probability distributions. Even after manual processing, the San Rafael data set is dense and consists of an admixture of events that can be categorised into Type A and Type B events, following the convention established by (Richardson and Jordan, 2002). For the purposes of probabilistic hazard assessments it is necessary to separate the Type $A$ and $B$ events to obtain unique probability distributions.

\section{Data}

San Rafael produces on average 10,000 manually accepted events a month and up to 20,000 in some months. Such a large and rich seismic data set is a two-edged sword. On the one hand, techniques such as passive source tomography depend on having many (fairly) uniformly distributed events. The different failure modes represented by different types of events also present alternative ways of probing the rock mass response to mining. On the other hand, the size of the data set presents challenges in data management and requires that careful reduction be applied in order to clarify these responses. The seismic event records considered here are those from events which occurred from 00:00:00 PET on 1 November 2010 to 00:00:00 PET on 1 February 2011. This results in a three month period giving similar time periods 
before and after the $M_{w} 2.0$ 2010/12/17, 07:17:09 PET event. We distinguish between two uses of the data, and this distinction leads to two different filtering criteria. The first use (order does not imply relative importance) is the conventional mine seismic historical and hazard assessment employing a seismic catalogue of event parameters, constructed from events which have been manually processed by a human operator.

This catalogue consists of the properties of those events tagged as valid ("accepted" in the vernacular), in the sense that the human operator judged the seismograms to be the results of rock fracturing (as opposed to blasts, mechanical vibration, and electronic noise or orepass vibration).

\section{$4 \quad$ Results}

\section{1 Passive source tomography}

The velocity distribution was modelled for three periods (1 to 26 November 2010, 27 November to 20 December 2010, and 21 December 2010 to 31 January 2011). The velocity models were computed using seismic tomography software designed and written by Advanced GeoSciences Imaging Solutions (AGSIS). Arrival times recorded at a minimum of four seismic sensors were used. Velocities were calculated at nodes and interpolated using a cubic spline, composed of 93 by 102 by 89 elements east-west by north-south by vertical, providing a grid resolution of $7.5 \mathrm{~m}$. Ten non-linear iterations involving ray tracing and inversion of the model parameters were performed to ensure convergence. Damping (the trade-off curve was used to select the appropriate damping parameters) and Gaussian smoothing were used to regularise the inversion. The smoothing kernel was set to $15 \mathrm{~m}$, twice the grid size. The resolution afforded by the inversion was measured using a standard checkerboard test.

Figure 3 presents P- and S-wave velocity snapshots. The images are slices (not projections) along strike, taken through a three dimensional block of results. Although the velocity has been calculated for the whole mine, only the structure in the vicinity of the major event and its variations are shown and discussed.

The changes in S-wave velocity are pronounced, while P-wave velocity varies only mildly throughout the period covered by this study. The snapshots of S-wave velocity that cover mainly the pre-event period (Figure 3(1) and 3(2)), show the presence of a high velocity anomaly nearly centred at the hypocenter of the major event. This high velocity region is surrounded by areas of lower velocities that are either below or above the average value of $3,150 \mathrm{~ms}^{-1}$. These two velocity snapshots do not significantly differ from each other suggesting that only minor change occurs in the fabric during the period pre-event period. The S-wave velocity snapshot of Figure 3(3) is, however, different from those of the two previous periods. The high velocity region is not apparent and is replaced by a low velocity anomaly that merges with two low velocity regions visible in the pre-event S-wave velocity snapshots. 

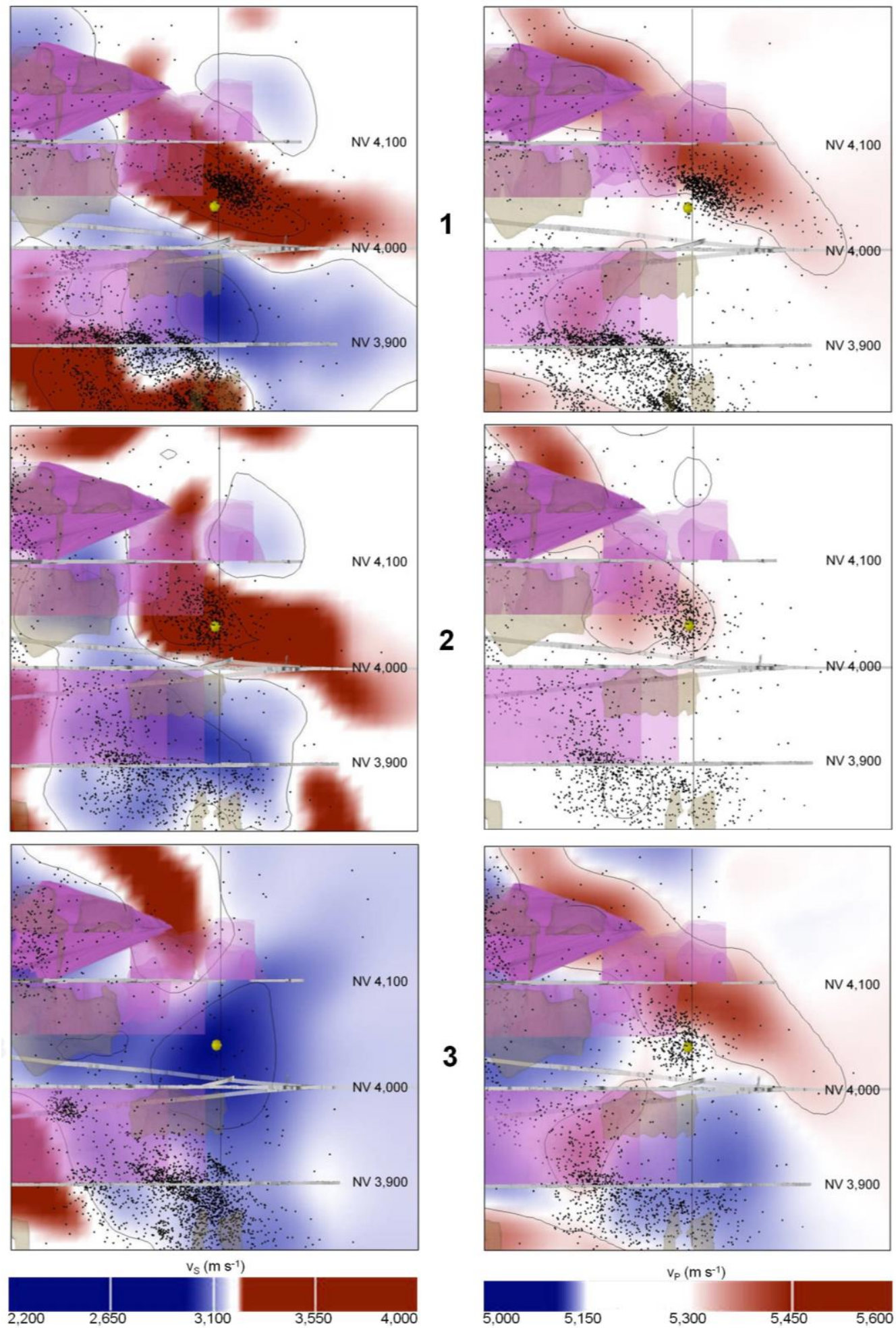

Figure $3 \mathrm{~S}$ - and P-wave velocity model snapshots for the three time periods extending from (1) 1-26 November 2010, (2) 27 November to 21 December 2010, and (3) 22 December 2010 to 31 January 2011. Images are long section slices through a three dimensional rendering of the velocity model. The location of the $\mathbf{M}_{\mathbf{w}} \mathbf{2}$.0 event is denoted by a yellow-gold symbol. Black dots represent the events used in the inversion 


\section{2 Spatial and temporal distribution of the microseismic activity}

\subsubsection{Type $A$ and Type $B$ events and clustering}

Any seismic event has a multi-dimensional nucleation volume within which conditions develop which leads to the event. The nucleation volume is spanned by time, spatial extent and source mechanism.

On a large scale, different source mechanisms result in at least two different populations of seismic events at blasting mines as evidenced by a multi-modal cumulative (tail) magnitude-frequency distribution (Figure 4, left). The Gutenberg-Richter relation, $\log _{10} \mathrm{~N}_{\geq m}=\mathrm{a}-\mathrm{bm}$, is fitted to the scaling regime of the distribution (in this paper, $\mathrm{m}=\mathrm{M}_{\mathrm{w}}$ - the Moment Magnitude) and the slope (b-value) is a scaling exponent which is linked to the mechanics of the earthquake process. Consistent probabilistic analysis requires that only events which contribute to a cumulative distribution with a unique critical exponent can be considered together. To date there are no exceptions to the observations that, for sets of small to intermediate magnitude events, $\mathrm{b} \approx 1.0 \pm 0.3$ (Ben-Zion, 2008). Type $\mathrm{A}(1.70<b<1.3)$ events can include seismic events due to fracturing of intact rock immediately after blasting, blasts, orepass noise and pillar crushing. Type $B$ events $(b \approx 1.0 \pm 0.3)$ are friction-dominated ruptures (slip-dominant) events. Hudyma (2008) reports a case in the literature where pillar and geological feature-related events were classed in a third category. Trifu et al. (1993) reports on non-unique distributions in a mine during a period when there was no production (i.e. no blasting), supporting the proposition that an interpretation of Type $A$ events as solely blast-related is not complete.

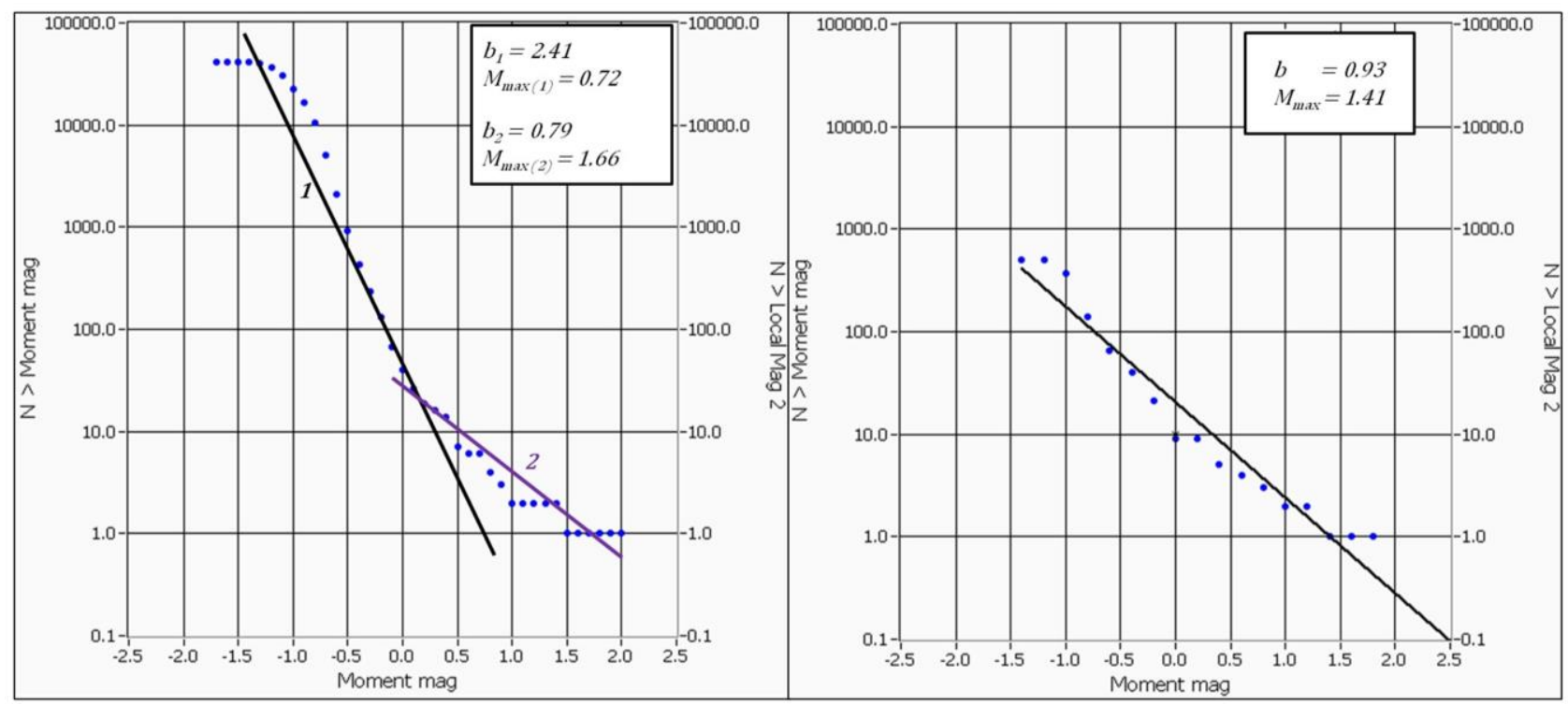

Figure 4 Left: Bimodal cumulative (tail) magnitude-frequency distribution characterised by two populations with different scaling exponents (b-values). Right: Frequency magnitude cumulative tail distribution of predominantly Type $B$ events after separation

It should therefore be emphasised that, for the purposes of a rock mechanical, physical interpretation, all events above $m_{\min }$ should be considered, because all types of events (with the exception of blasts and orepass noise) represent expressions of the stress redistribution within the rock mass.

For the purposes of probabilistic assessment, the Type $A$ and Type $B$ events are rapidly separated using the corner frequencies of event displacement spectra as a clustering measure. This separation is not perfect (Figure 4, right) but it does provide a quick means of establishing unique source volumes if followed by clustering the events using inter-event distances as a metric.

\section{2. 2 Trends in time - blast times and seismicity}

The $\mathrm{M}_{\mathrm{w}} 2.0$ 2010/12/17, 07:17:09 PET event was part of a cluster (denoted Cluster 06) of Type B events with a b-value of 0.93 . Type B events showed a qualitative cyclical behaviour, weakly correlated with 
blasting windows (04:00-05:00, 11:00-12:00, 19:00-20:00). The $M_{w} 2.0$ event occurred approximately three hours after the 04:00-05:00 blasting. There is no obvious relation between the Type $B$ event occurrence and the blasting rates at the mine. Note that the blasts were not located, hence the depiction of all events marked as blasts. In particular, the flurry of activity around the $M_{w} 2.0$ event does not appear to be closely linked to blasting. The total tonnage blasted in the five days before the event was similar or higher to other five-day periods, but most of it appeared to be concentrated on 15 December 2010.

\subsection{Trends in time - stress release and deformation}

In the cluster of interest, the time evolution of the median Energy Index showed a medium to long-term trend of decreasing stress release, while the Cumulative Apparent Volume (CAV) shows little indication of accelerating deformation prior to 17 December 2010 (Figure 5). The median Energy Index was calculated over two-and-a-half days and plotted every hour. Following a short period of scarcity in smaller events, suggestive of an onset of a change in conditions, there is some indication of a decreasing trend in shear stress changes at the seismic event sources as encoded in the Energy Index. There was little indication of accelerating deformation prior to the event (constant slope to the CAV). The event was followed by a twoweek consolidation period of low activity, notable for the scarcity of small events as stress was redistributed and in a fairly erratic fashion. We note that the Energy Index is influenced largely by smaller events. Finally, a period of recovery in the stress regime followed.

\section{Discussion}

For both analyses presented here, retention and use of more than just manually accepted ,"true", seismic event data is important. On the one hand, passive source tomography is critically dependent on data density. To this end it is important that all waveforms (not only those from manually accepted, "true", seismic events) are retained as long as they can provide reliable and consistent travel-times. We cannot overstress the fact that many types of sources illuminate the rock mass. This includes blasts, tensile fractures, mechanical noise and possibly orepass noise, but not electronic noise. On the other hand, for conventional analysis, it is sufficient to separate out only the blasts, orepass noise and mechanical noise. Both Type A and Type B events convey information about the state of stress of the rock mass at the locations of these events. It is only when seeking unambiguous probability functions (unique values) for hazard assessment that catalogues should be separated into Type A and Type B (and possibly Type C) events for separate but complementary analyses.

A seismic event does not have a single cause - the final failure in the rock is the end result of a combination of factors. These include geological (pre-existing planes of weakness such as faults, dykes, sills contacts), time (the process of removing rock transfers stress around the rock mass over days, months or years), production practices (this includes blast rates, tonnages and locations as well as mine geometry and sequencing which can lead to stressed pillars which can fail). Whereas the immediate cause of the $M_{w} 2.0$ 2010/12/17, 07:17:09 PET event was a strike slip failure in the rock, with an upwards component to the slip, underlying causes may have included the activation of a plane of weakness in the abutment almost perpendicular to the orebody by mining activities. The data shows that event was part of progressive failure on a north-south striking plane of weakness, dipping to the west at approximately $39^{\circ}$.

The orientation of the plane is almost perpendicular to that of the orebody. There are several distributions of events in the San Rafael data showing regular geometrical features (planar or ellipsoid). 

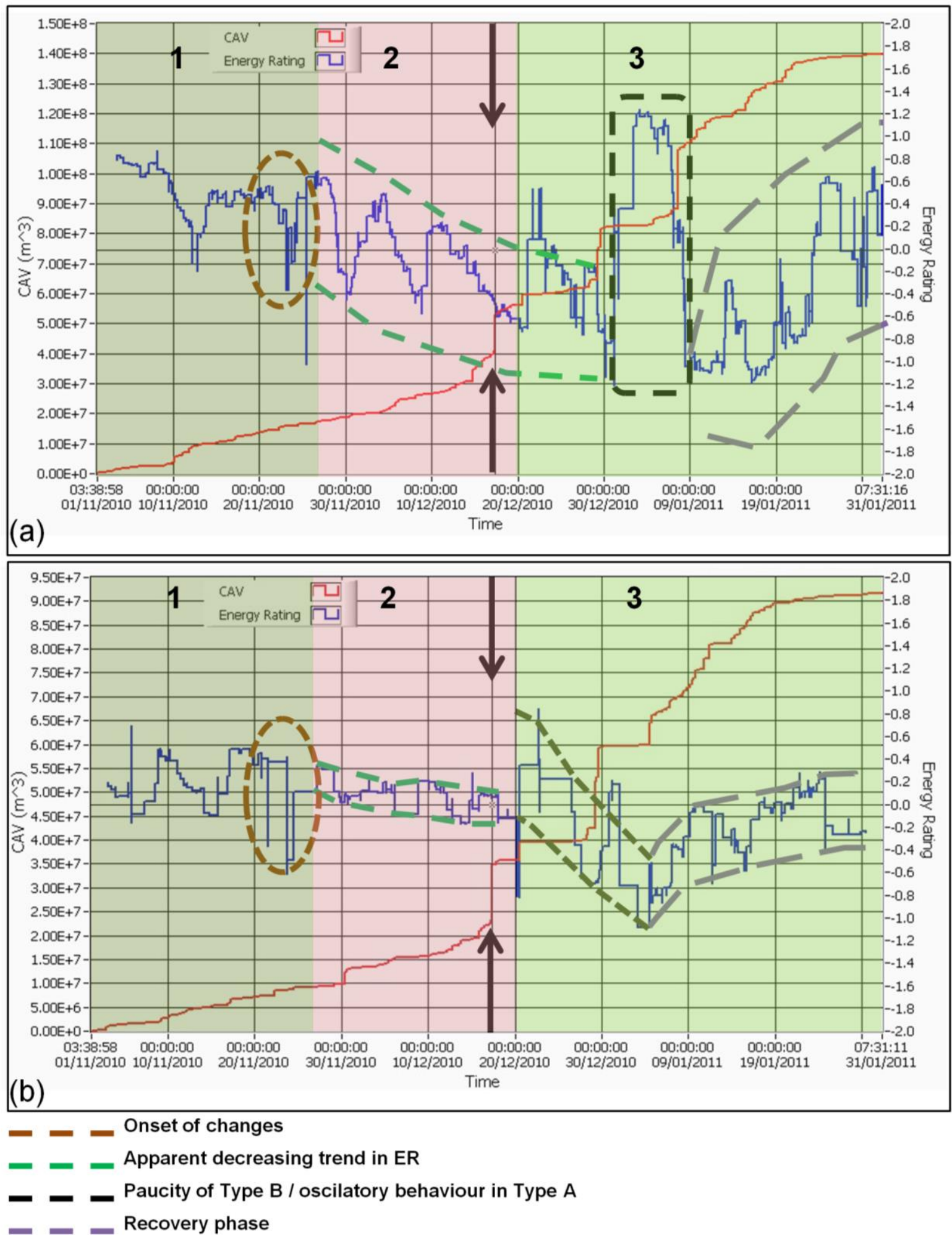

Figure 5 Time evolution of Energy Index and CAV (a) all events and (b) Type B events in Cluster 06, 1 November 2010 to 31 January 2011. Arrows indicate when the event occurred. The three coloured time windows correspond to the double-difference inversion windows

The final failure may have been in the sill pillar between NV4030 and NV4050, however, this failure could have been due to stress transfer from mining-induced convergence in the surrounding hangingwall and footwall over a period of at least six weeks as captured by the velocity modelling. It could be argued that the gap in blasting (13 and 14 December 2010, followed by a large shock on 15 December 2010), served to drive the rock mass system towards instability. In this way blasting may have contributed to the failures of 
17 December 2010. Although blasting may have precipitated failure, the mining geometry and sequence are the key drivers responsible for inducing the failure of the rock mass in the mining abutment and pillar.

As is pointed out in Hudyma (2008), it is common to attempt the use of a bouquet of techniques consisting of time series of quantities derived from seismic source parameters, plus time-lapse spatial distributions and probabilistic analyses to obtain some form of quantitative hazard indication. Trends of the type in Figure 5 are used by considering their similarity to laboratory experiments of the type in Guéguen et al. (2009) which relate acoustic emission, shear stress and strain in a sample. In microseismics, the Energy Index is a proxy for shear stress and CAV for strain. Guéguen et al. (2009) illustrates Bleurswiller sandstone exhibiting a critical pressure at which the seismic velocities show a sharp decrease. At this pressure, the porosity also shows a sharp irreversible decline. The critical pressure is the point at which grain crushing (microcracking) takes place. These experiments are part of the body of investigation into the characterisation of hydrocarbon reservoirs using time-lapse passive and active seismic measurements and, as such, focus on the rock types which host carbohydrate reservoirs. Given the felsic rock types at San Rafael, they serve only to prompt further discussion.

The relation between seismic velocity and stress has been known for decades. One could then conjecture that, prior to the event, stress accumulated in the rock mass in the area where the major event occurred, initially closing microfractures and consequently increasing the seismic velocities. Note that the decreasing median Energy Index (Figure 5) supports this as less and less stress is released seismically (a seismic wave is the sound of a fracture, after all). At a (rock type dependent) critical stress the formation of large fractures (large seismic events) initiate, cascading to the fracturing process of an aftershock sequence which also leads to a sharp reduction in the S-wave velocity.

Note that the median Energy Rating shows a sharp increase at (large energy radiation by a large event sequence) and immediately following the time of the large event.

\section{$6 \quad$ Concluding remarks}

The scaling of laboratory results to the field is neither clear nor trivial due to different scales of heterogeneity and different frequency responses; however, identification of velocity and stress in mines has been made before (Figure 6).

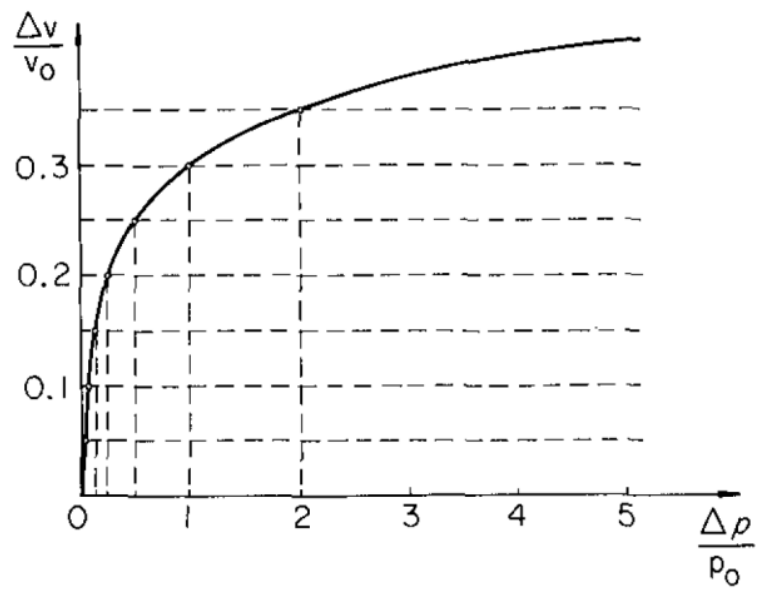

Figure 6 Variation in seismic velocity with pressure in a coal mine (from Körmendi et al., 1986)

In this paper, the dense and rich seismic catalogue at San Rafael is analysed in two ways. Conventional analysis was enabled by data reduction: separating the data into Type A and Type B events, and then performing spatial clustering in order to delineate a specific source of seismicity, believed to be connected to the occurrence of a large and damaging event. The separation of Type A and Type B events is done by clustering. The only purpose of this separation is to use unique probability distributions for probabilistic hazard assessments. 
Passive seismic tomography utilised all the data to model the velocity model in three time windows around the damaging event. The results of this analysis suggest that the high velocity region observed in the S-wave tomograms in the vicinity of the location of the major event Figure 3), prior to the event, is related to the concentration of stress in this area and that the transition from high velocity to low velocity after the event occurred is related to the release of seismic energy and fracturing of the rock. This result indicates that time lapse velocity modelling could help identify regions where stress accumulates that are consequently more prone to large scale seismic activity or rock burst. However, more case studies, including geological and structural characterisation and numerical stress modelling should be performed in order to establish the statistical significance of these patterns in different mining contexts.

It should be noted again that, from the perspective of passive tomography, all events (blasts, Type A, mechanical noise, excepting electronic noise and possibly orepass noise) in a catalogue serve to illuminate the rock mass. Microseismic databases should be managed with this in view, with care taken not to remove or delete Type A events or blasts.

\section{Acknow ledgements}

The authors are grateful to MINSUR S.A. for permission to submit this paper. Hannah Taylor and Nathan Currier (Golder Associates (NZ) Limited) proofread the manuscript.

\section{References}

Angus, D.A., Verdon, J.P., Fisher, Q.J. and Kendall, J-M. (2009) Exploring trends in microcrack properties of sedimentary rocks: An audit of dry-core velocity-stress measurements, Geophysics, Vol. 74, No. 5 (September-October 2009), pp. E293-E203.

Ben-Zion, Y. (2008) Collective behaviour of earthquakes and faults: Continuum-discrete transitions, progressive evolutionary changes, and different dynamic regimes, Reviews of Geophysics, Vol. 46, RG4006, doi:10.1029/2008RG000260.

Guéguen, Y., Sarout, J., Fortin, J. and Schubnel, A. (2009) Cracks in porous rocks: Tiny defects, strong effects, The Leading Edge, January 2009, pp. 40-47.

Hudyma, M.R. (2008) Analysis and interpretation of clusters of seismic events in mines, PhD thesis, Department of Civil and Resource Engineering, The University of Western Australia, November 2008, pp. 73-90.

Körmendi, A., Bodoky, T., Hermann, L., Dianiska, L. and Kàlmàn, T. (1986) Seismic measurements for safety in mines, Geophysical Prospecting, Vol. 34, pp. 1022-1037.

Richardson, E. and Jordan, T.H. (2002) Seismicity in Deep Gold Mines of South Africa: Implications for Tectonic Earthquakes, Bulletin of the Seismological Society of America, Vol. 92(5), pp. 1766-1782.

Trifu, C-I., Urbancic, T.I. and Young, R.P. (1993) Non-similar frequency-magnitude distribution for $m<1$ seismicity, Geophysical Research Letters, Vol. 20, pp. 427-430. 
Beyond coloured balls-making sense of large volumes of microseismic data with diverse

W. de Beer et al. source mechanisms 